\title{
Dynamics, Circuit Design, and Synchronization of a New Chaotic System with Closed Curve Equilibrium
}

\author{
Xiong Wang, ${ }^{1}$ Viet-Thanh Pham, ${ }^{2}$ and Christos Volos ${ }^{3}$ \\ ${ }^{1}$ Institute for Advanced Study, Shenzhen University, Shenzhen, Guangdong 518060, China \\ ${ }^{2}$ School of Electronics and Telecommunications, Hanoi University of Science and Technology, 01 Dai Co Viet, Hanoi, Vietnam \\ ${ }^{3}$ Department of Physics, Aristotle University of Thessaloniki, 54124 Thessaloniki, Greece \\ Correspondence should be addressed to Viet-Thanh Pham; pvt3010@gmail.com
}

Received 9 October 2016; Accepted 18 December 2016; Published 16 February 2017

Academic Editor: Ahmed G. Radwan

Copyright (C) 2017 Xiong Wang et al. This is an open access article distributed under the Creative Commons Attribution License, which permits unrestricted use, distribution, and reproduction in any medium, provided the original work is properly cited.

\begin{abstract}
After the report of chaotic flows with line equilibrium, there has been much attention to systems with uncountable equilibria in the past five years. This work proposes a new system with an infinite number of equilibrium points located on a closed curve. It is worth noting that the new system generates chaotic behavior as well as hidden attractors. Dynamics of the system with closed curve equilibrium have been investigated by using phase portraits, bifurcation diagram, maximal Lyapunov exponents, and Kaplan-York dimension. In addition, we introduce an electronic implementation of the theoretical system to verify its feasibility. Antisynchronization ability of the new system with infinite equilibria is studied by applying an adaptive control. This study suggests that there exist other chaotic systems with uncountable equilibria in need of further investigation.
\end{abstract}

\section{Introduction}

Although chaos has been an object of research since the 1960s, investigating chaotic systems is still a continuing concern within nonlinear dynamics field [1-7]. A great amount of different chaotic systems has been reported in the literature such as modified Lorenz chaotic system [8], jerk-based chaotic oscillators [9], three-dimensional (3D) system [10], $3 \mathrm{D}$ autonomous chaotic system with a single cubic nonlinearity [11], chaotic system with butterfly attractor [12], systems with multiscroll chaotic attractors [13-15], or fractional-order chaotic systems $[16,17]$. Moreover, the past decade has seen the rapid development of chaos-based applications in many areas from hardware pseudorandom number generators [18], autonomous mobile robots [19], MOS oscillators [20], encryption [21], chaotic masking communication [22], to information processing [23].

Recently, there has been renewed interest in chaotic systems with uncountable equilibria [24] (see the illustration in Figure 1). One of the most important discoveries is the finding of chaotic flows with line equilibrium [24-26]. The presence of parallel lines of equilibrium points [25] and perpendicular lines of equilibria [26] has also been studied.
Recent trends in systems with uncountable equilibria have led to systems with open curve equilibrium $[27,28]$ and closed curve equilibrium [29]. The issue of finding different shapes of closed curve equilibrium especially has received considerable critical attention [29-31]. Chaotic system with circular equilibrium has been obtained by using a search routine [29]. Gotthans and Petržela have constructed a chaotic flow with square equilibrium [29]. Chaotic system with uncountable equilibria located on a rounded square has been reported in [32]. From the computation point of view, it is now well established that systems with infinite equilibrium can be classified as special systems with "hidden attractor" [33-36], which have not treated much detail [37-39]. Discovering new chaotic systems with closed curve equilibrium is still an attractive research direction.

The main aim of this study is to investigate a novel chaotic system with closed-curve equilibrium. In Section 2, the model and the dynamics of the system are presented. Circuital implementation of the new system is reported in Section 3 while the ability of antisynchronization of such systems is discussed in Section 4. Finally, Section 5 gives conclusion remarks of our study. 


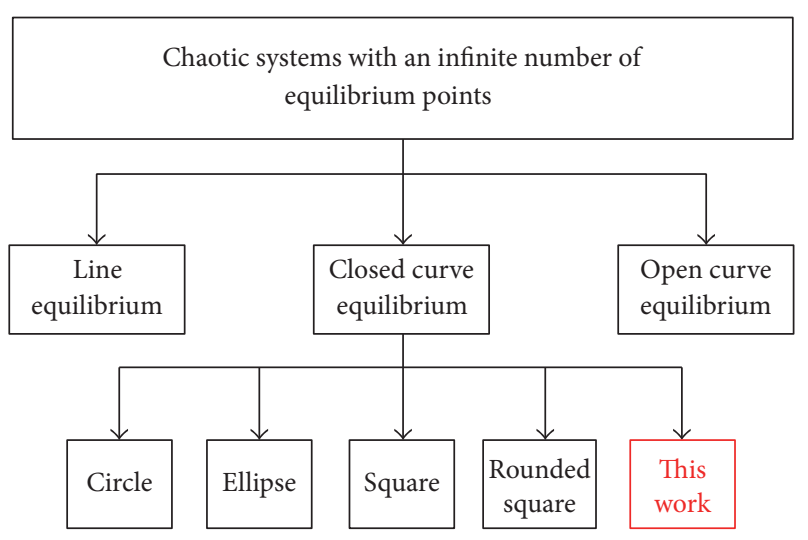

FIGURE 1: Systems with uncountable equilibria in which there has been an increasing interest in discovering different shapes of closed curve equilibrium.

\section{Description and Dynamics of the System with Closed Curve Equilibrium}

Recently, Gotthans et al. [30] have introduced chaotic systems with circle equilibrium and square equilibrium. Gotthans et al. system with circle equilibrium is given by

$$
\begin{aligned}
& \dot{x}=z, \\
& \dot{y}=-z\left(a y+b y^{2}+x z\right), \\
& \dot{z}=x^{2}+y^{2}-1,
\end{aligned}
$$

while the system with square equilibrium is described as

$$
\begin{aligned}
& \dot{x}=z, \\
& \dot{y}=-z(a y+b|y|)-x|z|, \\
& \dot{z}=|x|+|y|-1,
\end{aligned}
$$

in which $a$ and $b$ are constants. This investigation is significantly important because authors indicated the existence of chaotic systems with different shapes of equilibrium points [30]. Moreover, by generalizing the systems of Gotthans et al. we may get other new systems with an infinite number of equilibrium.

Based on the systems of Gotthans et al., we concentrate on a general model given by

$$
\begin{aligned}
& \dot{x}=z, \\
& \dot{y}=-z f_{1}(x, y, z), \\
& \dot{z}=f_{2}(x, y),
\end{aligned}
$$

in which three state variables are $x, y$, and $z$. The functions $f_{1}(x, y, z), f_{2}(x, y)$ are two arbitrary nonlinear functions. By solving $\dot{x}=0, \dot{y}=0$, and $\dot{z}=0$, it is straightforward to obtain

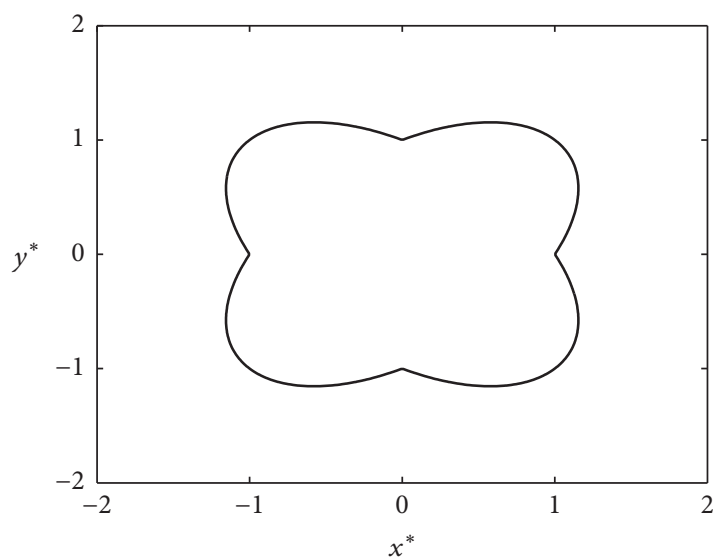

FIGURE 2: The special shape of equilibrium points in the new system (9)

the equilibrium point of general model (3). It means that we calculate

$$
\begin{aligned}
z & =0, \\
-z f_{1}(x, y, z) & =0, \\
f_{2}(x, y) & =0 .
\end{aligned}
$$

As can be seen from (4), we have $z=0$. By substituting $z=0$ into (5) and (6), it is easy to confirm that the equilibrium points of general model (3) are laid on the curve described by (6) in the plane $z=0$. Therefore numerous systems with closed curve equilibrium can be constructed by selecting appropriate functions $f_{1}(x, y, z)$ and $f_{2}(x, y)$.

In this work, we select two nonlinear functions $f_{1}(x, y, z)$ and $f_{2}(x, y)$ described by the following forms:

$$
\begin{aligned}
f_{1}(x, y, z) & =a y+b y^{2}+x z, \\
f_{2}(x, y) & =x^{2}-|x y|+y^{2}-1,
\end{aligned}
$$

in which $a$ and $b$ are two positive parameters. Substituting (7) and (8) into the general model (3), our new system is derived as

$$
\begin{aligned}
& \dot{x}=z, \\
& \dot{y}=-z\left(a y+b y^{2}+x z\right), \\
& \dot{z}=x^{2}-|x y|+y^{2}-1 .
\end{aligned}
$$

Combining (6) and (8), it is simple to verify that system (9) has an infinite number of equilibrium points $E\left(x^{*}, y^{*}, 0\right)$. Remarkably, such equilibrium points are described by

$$
\left(x^{*}\right)^{2}-\left|x^{*} y^{*}\right|+\left(y^{*}\right)^{2}=1 \text {. }
$$

In other words, the proposed system (9) has cloud-shaped curve equilibrium as illustrated in Figure 2. It is interesting to note that the cloud-shaped curve is different from basic shapes like circle, square, or ellipse [29-31]. Furthermore, 


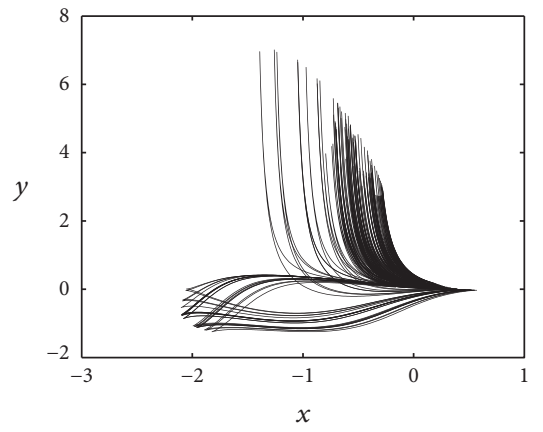

(a)

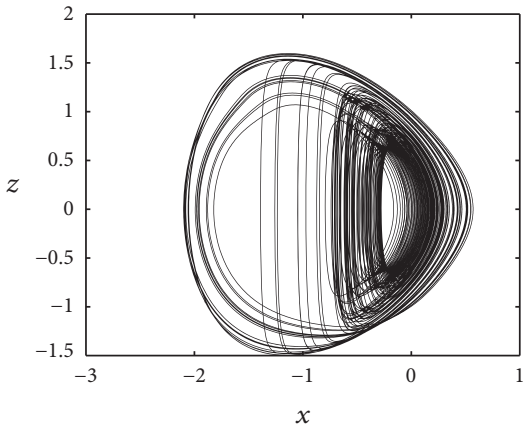

(b)

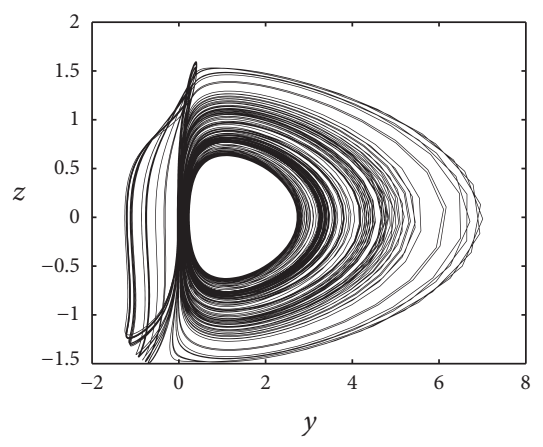

(c)

FIGURE 3: Projections of hidden chaotic attractors with cloud-shaped equilibrium in (a) $x-y$ plane, (b) $x-z$ plane, and (c) $y-z$ plane for $a=4$ and $b=2.5$.

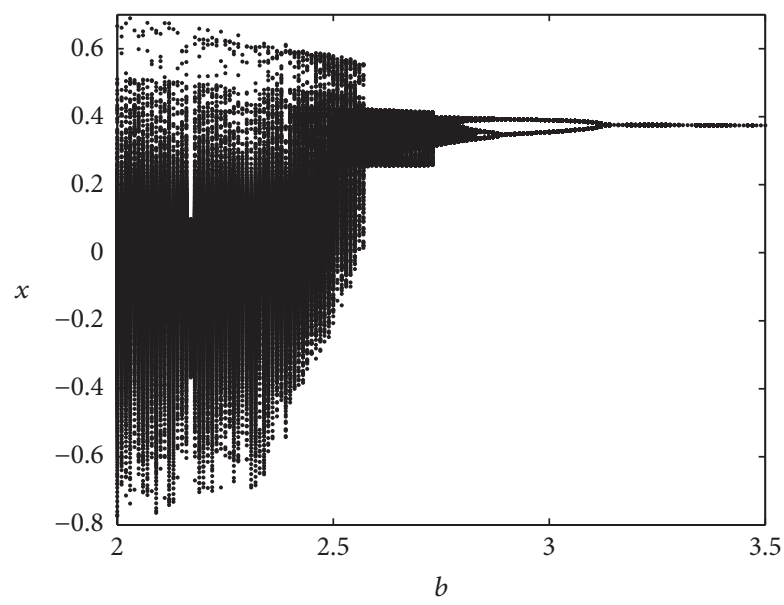

FIGURE 4: Bifurcation diagram of the system with closed curve equilibrium (9) when decreasing the value of the bifurcation parameter $b$ from 3.5 to 2 for $a=4$.

system (9) belongs to a rare class of systems with "hidden attractors" [39]. Therefore the investigation of system (9) will enhance our understanding of systems with "hidden attractors" which is an increasingly important area in practical engineering [40-42].

It is worth noting that system (9) with infinite equilibria displays chaotic attractors (see Figure 3 ) for $a=4, b=2.5$ and the initial conditions $(x(0), y(0), z(0))=(0.01,0.02,0.01)$. Chaotic behavior of the system is confirmed by the Lyapunov exponents $L_{1}=0.13>0, L_{2}=0$, and $L_{3}=-0.6853$. The well-known Wolf et al's method has been used to calculate the Lyapunov exponents [43] and the time of computation is 10,000 . In this case, the corresponding Kaplan-York dimension of system (9) is $D_{\mathrm{KY}}=2.1897$. It is noted that unexpected jumps in the values of the local Lyapunov exponents and Lyapunov dimension may occur. Thus the infimum over time interval often gives better estimates. In addition, it is difficult to get the same values of the finite-time local Lyapunov exponents and Lyapunov dimension for different points. Therefore the maximum of the finite-time local Lyapunov dimensions on the grid of point has to be considered [44-46].

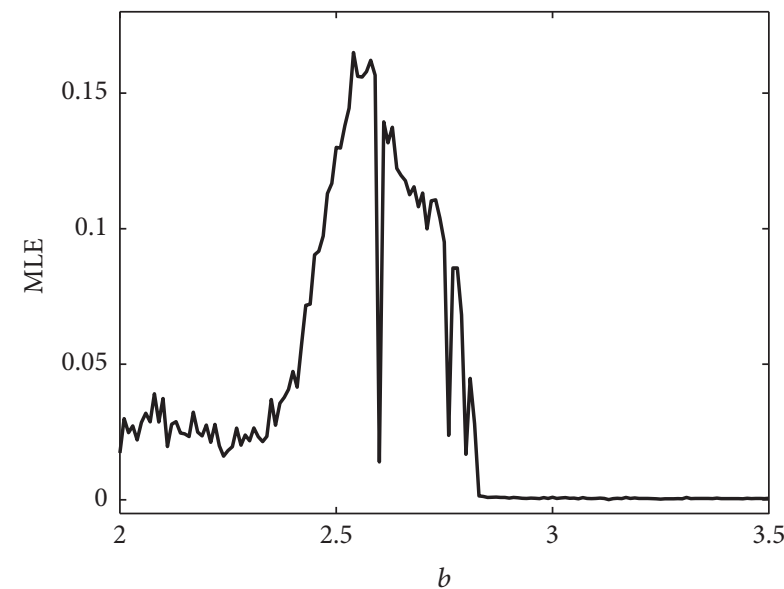

FIGURE 5: Maximal Lyapunov exponents of the system with closed curve equilibrium (9) in the range $2 \leq b \leq 3.5$ for $a=4$.

By changing the value of the parameter $b$, the dynamical properties of the system with infinite equilibria (9) are able to be discovered. Figures 4 and 5 display the bifurcation diagram and the diagram of maximal Lyapunov exponents of system (9), respectively. As can be seen from Figures 4 and 5, there is the presence of the classical period doubling route to chaos when decreasing the value of the bifurcation parameter $b$. The system with cloud-shaped equilibrium generate periodical states in the range of $2.83 \leq b \leq 3.5$. For instance, system (9) can display the period-1 state for $b=3.25$ (see Figure 6(a)) and the period-2 state for $b=2.95$ (see Figure 6(b)). For $b<2.83$, chaotic states are able to be observed in system (9).

\section{Circuit Design of the New System with Closed Curve Equilibrium}

In order to illustrate the feasibility of the theoretical system with a closed curve of equilibrium points (9), we present its circuital implementation in this section. For the sake of simplicity, we applied the design approach based on operational amplifiers [47-49]. It is noting that this design approach is not complex and requires common electronic elements only [13, 


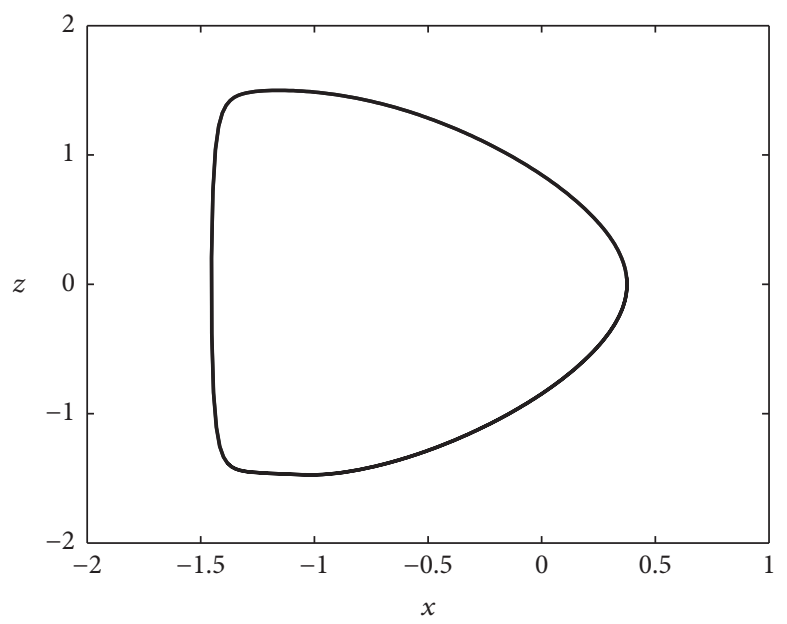

(a)

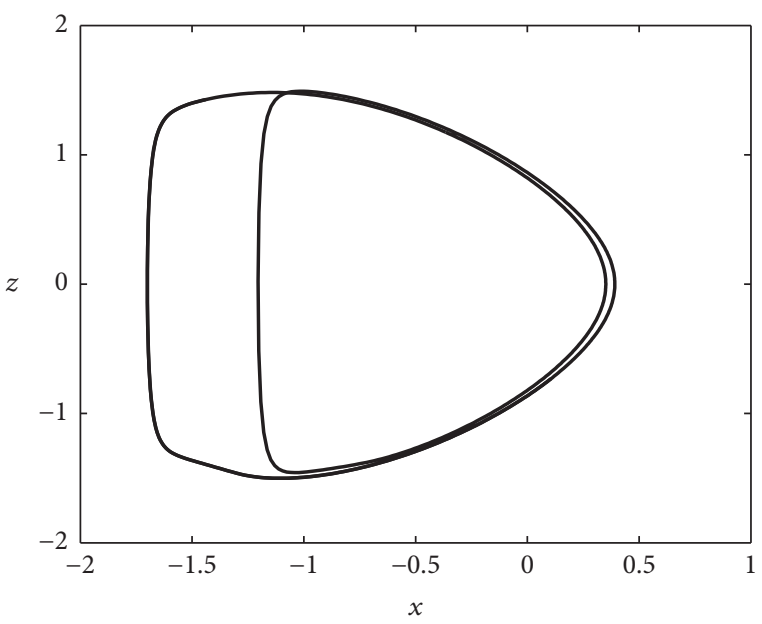

(b)

Figure 6: Periodical behavior of system (9) represented in the $x-z$ plane: (a) period-1 state $(b=3.25)$ and $(b)$ period -2 state $(b=2.95)$.

50]. Our designed circuit is shown in Figure 7, in which there are three integrators $\left(U_{1}-U_{3}\right)$ with corresponding output voltages $\left(v_{C_{1}}-v_{C_{3}}\right)$. The absolute nonlinearity is realized by the circuitry including two operational amplifiers $\left(U_{4}, U_{5}\right)$ and two diodes $\left(D_{1}, D_{2}\right)$.

It is simple to derive the circuital equation of the circuit in Figure 7:

$$
\begin{aligned}
& \frac{d v_{C_{1}}}{d t}=\frac{1}{R C_{1}}\left(\frac{R}{R_{1}} v_{C_{3}}\right), \\
& \frac{d v_{C_{2}}}{d t}=\frac{1}{R C_{2}}\left(-\frac{R}{R_{2} 10 V} v_{C_{2}} v_{C_{3}}\right. \\
&\left.-\frac{R}{R_{3} 10^{2} V^{2}} \frac{R_{9}+R_{10}}{R_{9}} v_{C_{2}}^{2} v_{C_{3}}-\frac{R}{R_{4} 10^{2} V^{2}} v_{C_{1}} v_{C_{3}}^{2}\right), \\
& \frac{d v_{C_{3}}}{d t}=\frac{1}{R C_{3}}\left(\frac{R}{R_{5} 10 V} v_{C_{1}}^{2}-\frac{R}{R_{6} 10 V}\left|v_{C_{1}} v_{C_{2}}\right|\right. \\
&\left.\quad+\frac{R}{R_{7} 10 V} v_{C_{2}}^{2}-\frac{R}{R_{8}} V_{1}\right) .
\end{aligned}
$$

The dimensionless system (12) is obtained by normalizing the circuital equation with $\tau=t / R C$, that is,

$$
\begin{aligned}
& \dot{X}=\frac{R}{R_{1}} Z, \\
& \dot{Y}=-\frac{R}{10 R_{2}} Y Z-\frac{R}{10^{2} R_{3}} \frac{R_{9}+R_{10}}{R_{9}} Y^{2} Z-\frac{R}{10^{2} R_{4}} X Z^{2}, \\
& \dot{Z}=\frac{R}{10 R_{5}} X^{2}-\frac{R}{10 R_{6}}|X Y|+\frac{R}{10 R_{7}} Y^{2}-\frac{R}{R_{8}} \frac{V_{1}}{1 V} .
\end{aligned}
$$

As can be seen in (12), the state variables $(X, Y, Z)$ are equivalent to the voltages of capacitors $\left(v_{C_{1}}, v_{C_{2}}, v_{C_{3}}\right)$. The dimensionless system (12) corresponds to the proposed system (9) with $a=R / 10 R_{2}$ and $b=\left(R / 10^{2} R_{3}\right)\left(\left(R_{9}+R_{10}\right) / R_{9}\right)$.

We select the values of electronic components to realize the theoretical systems (9) for $a=4$ and $b=2.5$ as follows:
$R_{1}=R_{8}=R=100 \mathrm{k} \Omega, R_{2}=2.5 \mathrm{k} \Omega, R_{3}=4 \mathrm{k} \Omega$, $R_{4}=1 \mathrm{k} \Omega, R_{0}=R_{5}=R_{6}=R_{7}=10 \mathrm{k} \Omega, R_{9}=11 \mathrm{k} \Omega$, $R_{10}=99 \mathrm{k} \Omega, V_{1}=1 \mathrm{~V}_{\mathrm{DC}}$, and $C_{1}=C_{2}=C_{3}=$ $C=4.7 \mathrm{nF}$. The PSpice projections of chaotic attractors with infinite equilibria are presented in Figure 8. From Figure 8 we can see that the designed circuit displays chaotic signals. The PSpice results also indicate that the circuit can emulate the theoretical model (9). It is necessary to remark that results of circuit simulation depend on the discretization step. As a result, we should consider the discretization step seriously when simulating electronic circuits, especially in the case of hidden oscillations [51,52]. Moreover, it is worth noting that realizing the circuit with real analog devices is better than realizing the circuit in PSpice. Therefore, realizing the circuit with real analog devices will be our next work.

\section{Antisynchronization of the Identical Systems with Infinite Equilibria}

After the investigation of Pecora and Carrol related to synchronization in chaotic systems [53], various synchronization techniques were studied extensively, for example, global chaos synchronization [54], hybrid synchronization [55], ragged synchronizability [56], and so on [57]. Remarkably, the possibility of synchronization of two identical chaotic systems has received considerable attention due to the vital role in practical applications $[57,58]$. In this section, we discover the antisynchronization of two new systems with cloud-shaped equilibrium, named the master system and the slave system.

We consider the master system with closed curve equilibrium

$$
\begin{aligned}
& \dot{x}_{1}=z_{1}, \\
& \dot{y}_{1}=-a y_{1} z_{1}-b y_{1}^{2} z_{1}-x_{1} z_{1}^{2}, \\
& \dot{z}_{1}=x_{1}^{2}-\left|x_{1} y_{1}\right|+y_{1}^{2}-1,
\end{aligned}
$$



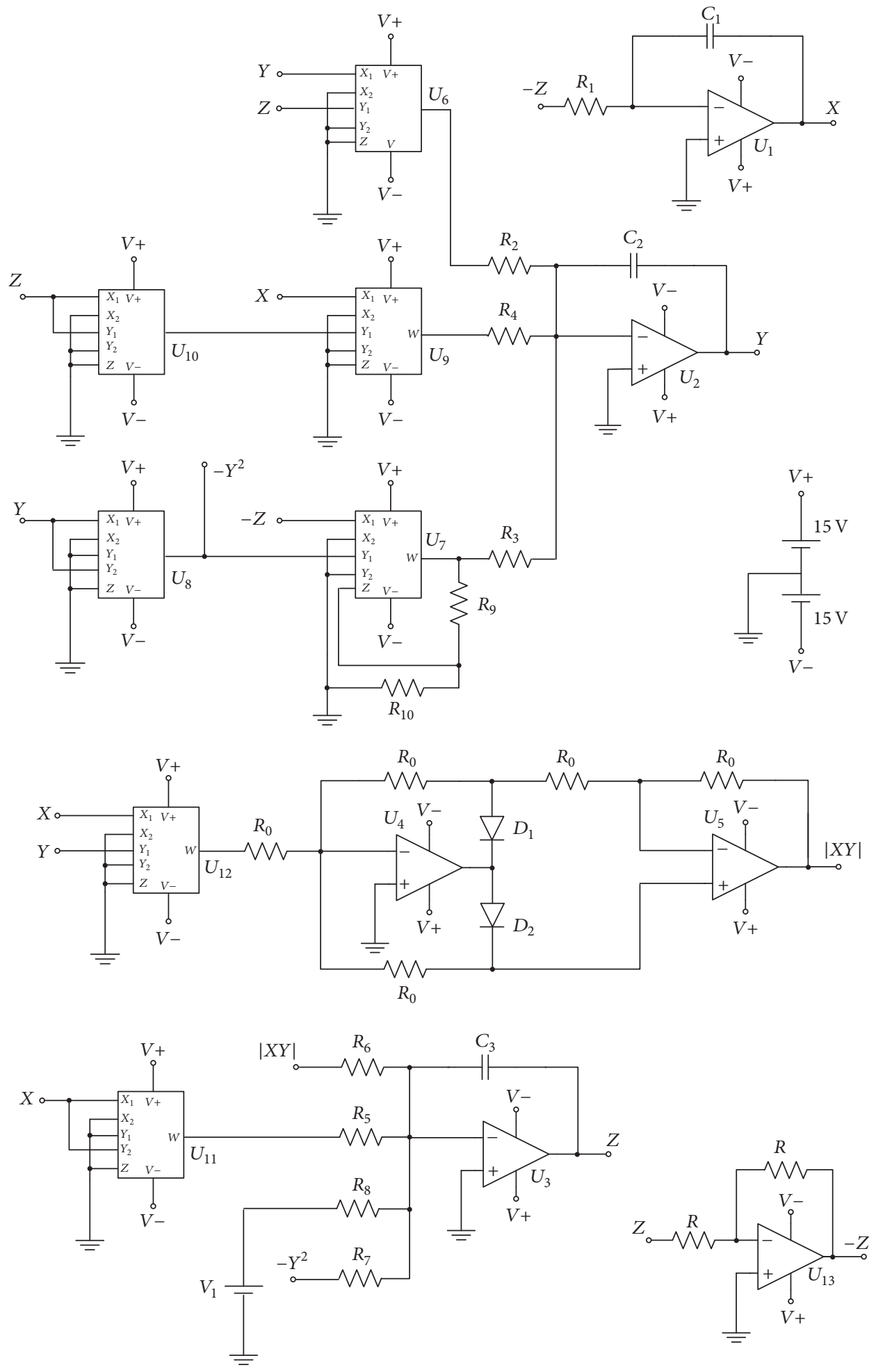

Figure 7: The schematic of the circuit which realizes the theoretical chaotic system with closed curve equilibrium (9). Here the values of three capacitors are chosen as $C_{1}=C_{2}=C_{3}=C=4.7 \mathrm{nF}$. 


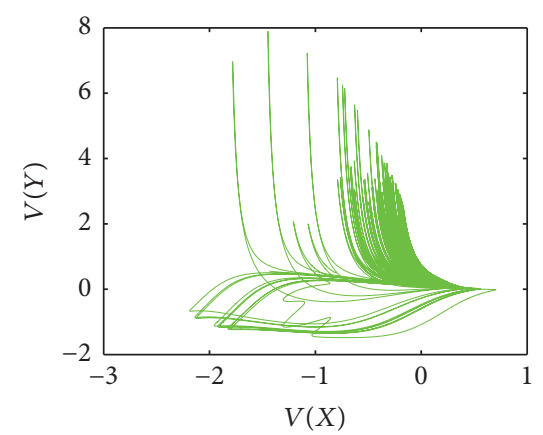

(a)

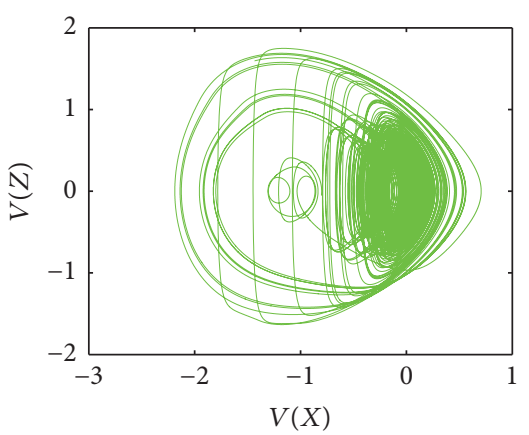

(b)

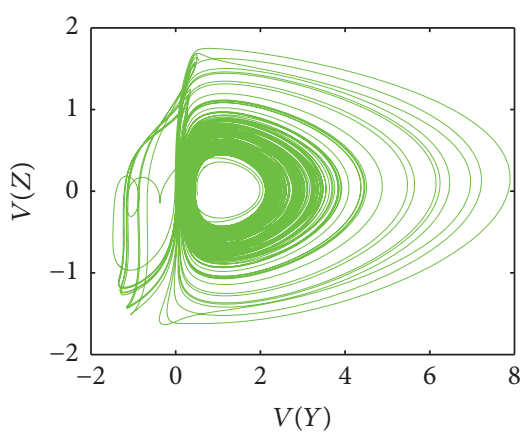

(c)

FIgURE 8: PSpice chaotic attractors of the circuit with cloud-shaped equilibrium in (a) $X-Y$ plane, (b) $X-Z$ plane, and (c) $Y-Z$ plane.

in which three state variables are $x, y$, and $z$ while $a$ and $b$ are two unknown parameters.

The slave system is described by

$$
\begin{aligned}
& \dot{x}_{2}=z_{2}+u_{x}, \\
& \dot{y}_{2}=-a y_{2} z_{2}-b y_{2}^{2} z_{2}-x_{2} z_{2}^{2}+u_{y}, \\
& \dot{z}_{2}=x_{2}^{2}-\left|x_{2} y_{2}\right|+y_{2}^{2}-1+u_{z},
\end{aligned}
$$

in which the adaptive control is $\mathbf{u}=\left[u_{x}, u_{y}, u_{z}\right]^{T}$.

To indicate the difference between the slave system (14) and the master system (13), the state errors are defined as

$$
\begin{aligned}
& e_{x}=x_{2}+x_{1}, \\
& e_{y}=y_{2}+y_{1}, \\
& e_{z}=z_{2}+z_{1} .
\end{aligned}
$$

Similarly, we calculate the parameter estimation errors

$$
\begin{aligned}
& e_{a}=a-\widehat{a}, \\
& e_{b}=b-\widehat{b},
\end{aligned}
$$

in which $\widehat{a}, \hat{b}$ are the estimations of two unknown parameters $a, b$, respectively. It is trivial to get the dynamics of the parameter estimation errors:

$$
\begin{aligned}
& \dot{e}_{a}=-\dot{\hat{a}} \\
& \dot{e}_{b}=-\dot{\hat{b}} .
\end{aligned}
$$

The aim of our work is to get the antisynchronization between the slave system and the master system; therefore, the adaptive control is proposed by

$$
\begin{aligned}
u_{x}= & -e_{z}-k_{x} e_{x}, \\
u_{y}= & \widehat{a}\left(y_{1} z_{1}+y_{2} z_{2}\right)+\widehat{b}\left(y_{1}^{2} z_{1}+y_{2}^{2} z_{2}\right) \\
& +\left(x_{1} z_{1}^{2}+x_{2} z_{2}^{2}\right)-k_{y} e_{y}, \\
u_{z}= & -x_{1}^{2}-x_{2}^{2}+\left|x_{1} y_{1}\right|+\left|x_{2} y_{2}\right|-y_{1}^{2}-y_{2}^{2}+2-k_{z} e_{z} .
\end{aligned}
$$

In (18), three positive gain constants are $k_{x}, k_{y}$, and $k_{z}$ while the parameter update law is defined by

$$
\begin{aligned}
& \dot{\hat{a}}=-e_{y}\left(y_{1} z_{1}+y_{2} z_{2}\right), \\
& \dot{\hat{b}}=-e_{y}\left(y_{1}^{2} z_{1}+y_{2}^{2} z_{2}\right) .
\end{aligned}
$$

The antisynchronization of the slave system (14) and the master system (13) is verified by applying Lyapunov stability theory [59] as follows.

We select the Lyapunov function described by

$$
V\left(e_{x}, e_{y}, e_{z}, e_{a}, e_{b}\right)=\frac{1}{2}\left(e_{x}^{2}+e_{y}^{2}+e_{z}^{2}+e_{a}^{2}+e_{b}^{2}\right)
$$

Therefore, the differentiation of the Lyapunov function is given by

$$
\dot{V}=e_{x} \dot{e}_{x}+e_{y} \dot{e}_{y}+e_{z} \dot{e}_{z}+e_{a} \dot{e}_{a}+e_{b} \dot{e}_{b} .
$$

Combining (13)-(16) and (18), we get

$$
\begin{aligned}
& \dot{e}_{x}=-k_{x} e_{x} \\
& \dot{e}_{y}=-e_{a}\left(y_{1} z_{1}+y_{2} z_{2}\right)-e_{b}\left(y_{1}^{2} z_{1}+y_{2}^{2} z_{2}\right)-k_{y} e_{y}, \\
& \dot{e}_{z}=-k_{z} e_{z} .
\end{aligned}
$$

By substituting (17) and (22) into (21), the differentiation of $V$ is rewritten in the form

$$
\dot{V}=-k_{x} e_{x}^{2}-k_{y} e_{y}^{2}-k_{z} e_{z}^{2}
$$

Obviously, the differentiation of the Lyapunov function $\dot{V}$ is a negative semidefinite function. As a result, it is simple to verify $e_{x} \rightarrow 0, e_{y} \rightarrow 0$, and $e_{z} \rightarrow 0$ exponentially as $t \rightarrow \infty$ based on Barbalat's lemma [59]. The complete antisynchronization between the master system and the slave system is proved.

We take an example to illustrate the calculation of the antisynchronization scheme. The parameter values of the master system and slave system are fixed as

$$
\begin{aligned}
& a=4, \\
& b=2.5 .
\end{aligned}
$$




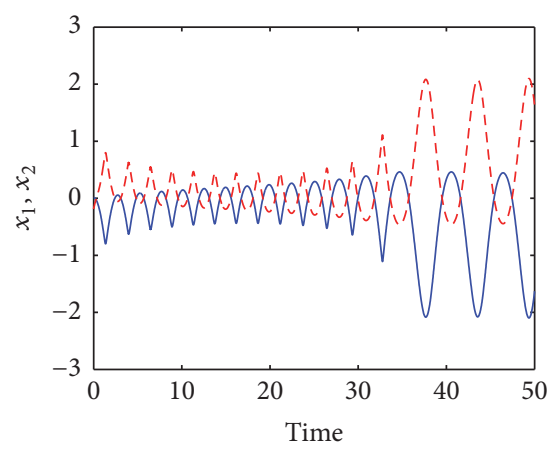

(a)

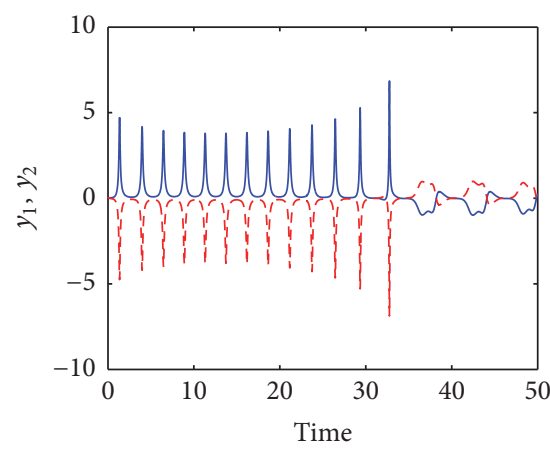

(b)

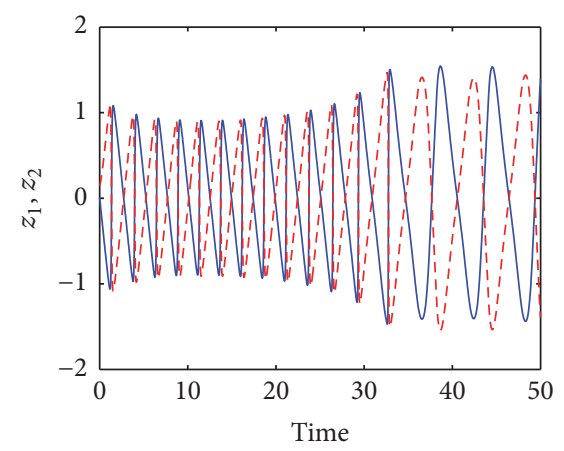

(c)

FIGURE 9: Antisynchronization of the slave system and the master system (a) $x_{1}, x_{2}$ state variables, (b) $y_{1}, y_{2}$ state variables, and (c) $z_{1}, z_{2}$ state variables (state variables of the slave system (red dashed lines), state variables of the master system (blue solid lines)).

We assume that the initial states of the master system (13) are

$$
\begin{aligned}
& x_{1}(0)=0.01, \\
& y_{1}(0)=0.02, \\
& z_{1}(0)=0.01,
\end{aligned}
$$

while the initial states of the slave system (14) are taken as

$$
\begin{aligned}
& x_{2}(0)=-0.19, \\
& y_{2}(0)=0.07, \\
& z_{2}(0)=0.15 .
\end{aligned}
$$

The positive gain constants are chosen as $k_{x}=5, k_{y}=5$, and $k_{z}=5$. For numerical simulations, we take the initial condition of the parameter estimate as

$$
\begin{aligned}
& \widehat{a}(0)=4.1, \\
& \widehat{b}(0)=2.6 .
\end{aligned}
$$

It is apparent from Figure 9 that there is antisynchronization of the respective states of the systems with cloud-shaped equilibrium (13) and (14). The time-history of the synchronization errors $e_{x}, e_{y}$, and $e_{z}$ is shown in Figure 10. It is straightforward to verify that Figure 10 depicts the antisynchronization of the master and slave systems.

\section{Conclusions}

The main goal of the current study is to propose a novel unusual system with infinite number of equilibrium points which lay on a closed curve. Dynamics of the new system have been investigated via different tools such as phase portrait, bifurcation diagram, Kaplan-York dimension, and maximal Lyapunov exponents. The feasibility of the theoretical model is clearly verified by the circuit implementation. In addition, the research also shows that antisynchronization of systems with closed curve equilibrium is obtained by introducing an adaptive control. The findings in this study provide a new understanding of system with infinite equilibria. In terms

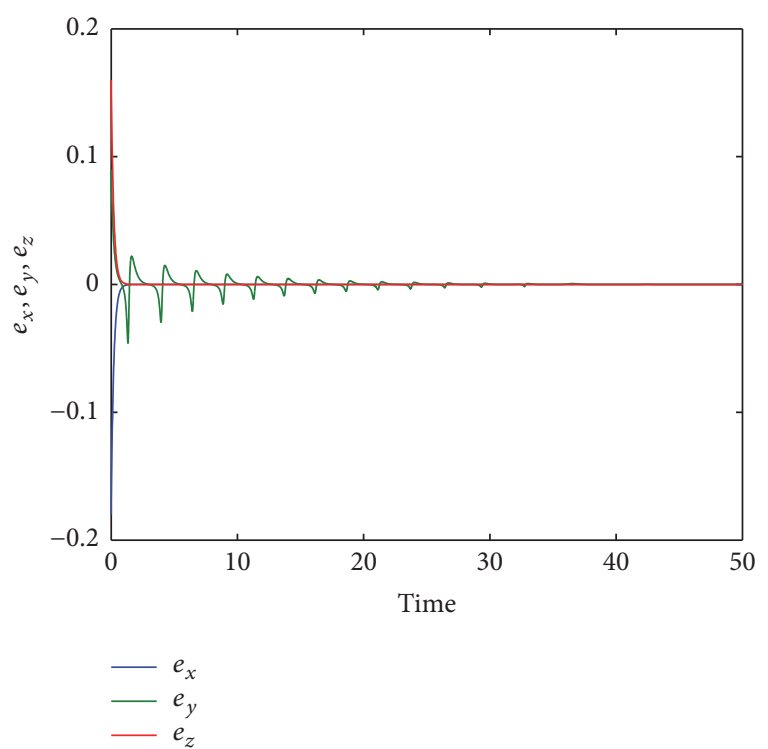

FIGURE 10: Time-history of the antisynchronization errors between the systems with cloud-shaped equilibrium (13) and (14).

of directions for future research, further work could explore chaos-based applications of such new system. For example, chaotic behaviors of other systems which are similar to the new one are useful for generating hardware pseudorandom number [18], controlling motions of autonomous mobile robots [19], or using in secure communications [22].

\section{Competing Interests}

The authors declare that there is no conflict of interests regarding the publication of this paper.

\section{Acknowledgments}

The authors acknowledge Professor GuanRong Chen, Department of Electronic Engineering, City University of Hong Kong, for suggesting many helpful references. The author Xiong Wang was supported by the National Natural 
Science Foundation of China (nos. 11547117 and 61601306) and Shenzhen Overseas High Level Talent Peacock Project Fund (no. 20150215145C).

\section{References}

[1] E. N. Lorenz, "Deterministic nonperiodic flow," Journal of the Atmospheric Sciences, vol. 20, no. 2, pp. 130-141, 1963.

[2] G. R. Chen and T. Ueta, "Yet another chaotic attractor," International Journal of Bifurcation and Chaos, vol. 9, no. 7, pp. 1465-1466, 1999.

[3] J. C. Sprott, "Some simple chaotic flows," Physical Review E, vol. 50, no. 2, pp. R647-R650, 1994.

[4] J. C. Sprott, Elegant Chaos Algebraically Simple Chaotic Flows, World Scientific, Singapore, 2010.

[5] M. P. Aghababa and M. Borjkhani, "Chaotic fractional-order model for muscular blood vessel and its control via fractional control scheme," Complexity, vol. 20, no. 2, pp. 37-46, 2014.

[6] F. Zhang, C. Mu, G. Zhang, and D. Lin, "Dynamics of two classes of Lorenz-type chaotic systems," Complexity, vol. 21, no. 1, pp. 363-369, 2015.

[7] A. T. Azar and S. Vaidyanathan, Advances in Chaos Theory and Intelligent Control, Springer, Berlin, Germany, 2016.

[8] A. G. Radwan, A. M. Soliman, and A. El-Sedeek, "MOS realization of the modified Lorenz chaotic system," Chaos, Solitons and Fractals, vol. 21, no. 3, pp. 553-561, 2004.

[9] A. S. Mansingka, M. A. Zidan, M. L. Barakat, A. G. Radwan, and K. N. Salama, "Fully digital jerk-based chaotic oscillators for high throughput pseudo-random number generators up to 8.77 Gbits/s," Microelectronics Journal, vol. 44, no. 9, pp. 744752, 2013.

[10] S. Vaidyanathan, "Analysis and adaptive synchronization of eight-term 3-D polynomial chaotic systems with three quadratic nonlinearities," European Physical Journal: Special Topics, vol. 223, no. 8, pp. 1519-1529, 2014.

[11] V. Sundarapandian and I. Pehlivan, "Analysis, control, synchronization, and circuit design of a novel chaotic system," Mathematical and Computer Modelling, vol. 55, no. 7-8, pp. 1904-1915, 2012.

[12] I. Pehlivan, I. M. Moroz, and S. Vaidyanathan, "Analysis, synchronization and circuit design of a novel butterfly attractor," Journal of Sound and Vibration, vol. 333, no. 20, pp. 5077-5096, 2014.

[13] A. Akgul, I. Moroz, I. Pehlivan, and S. Vaidyanathan, "A new four-scroll chaotic attractor and its engineering applications," Optik, vol. 127, no. 13, pp. 5491-5499, 2016.

[14] A. G. Radwan, A. M. Soliman, and A. S. Elwakil, "1-D digitallycontrolled multiscroll chaos generator," International Journal of Bifurcation and Chaos, vol. 17, no. 1, pp. 227-242, 2007.

[15] M. A. Zidan, A. G. Radwan, and K. N. Salama, "Controllable Vshape multiscroll butterfly attractor: system and circuit implementation," International Journal of Bifurcation and Chaos, vol. 22, no. 6, Article ID 1250143, 2012.

[16] A. Hajipour and H. Tavakoli, "Analysis and circuit simulation of a novel nonlinear fractional incommensurate order financial system," Optik, vol. 127, no. 22, pp. 10643-10652, 2016.

[17] E. Zambrano-Serrano, E. Campos-Cantón, and J. M. MunozPacheco, "Strange attractors generated by a fractional order switching system and its topological horseshoe," Nonlinear Dynamics, vol. 83, no. 3, pp. 1629-1641, 2016.
[18] M. L. Barakat, A. S. Mansingka, A. G. Radwan, and K. N. Salama, "Generalized hardware post-processing technique for chaos-based pseudorandom number generators," ETRI Journal, vol. 35, no. 3, pp. 448-458, 2013.

[19] C. K. Volos, I. M. Kyprianidis, and I. N. Stouboulos, "A chaotic path planning generator for autonomous mobile robots," Robotics and Autonomous Systems, vol. 60, no. 4, pp. 651-656, 2012.

[20] A. G. Radwan, A. M. Soliman, and A.-L. El-Sedeek, "An inductorless CMOS realization of Chua's circuit," Chaos, Solitons \& Fractals, vol. 18, no. 1, pp. 149-158, 2003.

[21] C. K. Volos, I. M. Kyprianidis, and I. N. Stouboulos, "Image encryption process based on chaotic synchronization phenomena," Signal Processing, vol. 93, no. 5, pp. 1328-1340, 2013.

[22] S. Çiçek, A. Ferikoğlu, and I. Pehlivan, "A new 3D chaotic system: dynamical analysis, electronic circuit design, active control synchronization and chaotic masking communication application," Optik, vol. 127, no. 8, pp. 4024-4030, 2016.

[23] V. E. Bondarenko, "Information processing, memories, and synchronization in chaotic neural network with the time delay," Complexity, vol. 11, no. 2, pp. 39-52, 2005.

[24] S. Jafari and J. C. Sprott, "Simple chaotic flows with a line equilibrium," Chaos, Solitons \& Fractals, vol. 57, pp. 79-84, 2013.

[25] C. Li and J. C. Sprott, "Chaotic flows with a single nonquadratic term,” Physics Letters A, vol. 378, no. 3, pp. 178-183, 2014.

[26] C. Li, J. C. Sprott, Z. Yuan, and H. Li, "Constructing chaotic systems with total amplitude control," International Journal of Bifurcation and Chaos, vol. 25, no. 10, Article ID 1530025, 2015.

[27] Y. Chen and Q. Yang, "A new Lorenz-type hyperchaotic system with a curve of equilibria," Mathematics and Computers in Simulation, vol. 112, pp. 40-55, 2015.

[28] V.-T. Pham, S. Jafari, C. Volos, S. Vaidyanathan, and T. Kapitaniak, "A chaotic system with infinite equilibria located on a piecewise linear curve," Optik, vol. 127, no. 20, pp. 9111-9117, 2016.

[29] T. Gotthans and J. Petržela, "New class of chaotic systems with circular equilibrium," Nonlinear Dynamics, vol. 81, no. 3, pp. 1143-1149, 2015.

[30] T. Gotthans, J. C. Sprott, and J. Petrzela, "Simple chaotic flow with circle and square equilibrium," International Journal of Bifurcation and Chaos, vol. 26, no. 8, Article ID 1650137, 2016.

[31] V.-T. Pham, S. Jafari, X. Wang, and J. Ma, "A chaotic system with different shapes of equilibria," International Journal of Bifurcation and Chaos, vol. 26, no. 4, Article ID 1650069, 5 pages, 2016.

[32] V.-T. Pham, S. Jafari, C. Volos, A. Giakoumis, S. Vaidyanathan, and T. Kapitaniak, "A chaotic system with equilibria located on the rounded square loop and its circuit implementation," IEEE Transactions on Circuits and Systems II: Express Briefs, vol. 63, no. 9, pp. 878-882, 2016.

[33] G. A. Leonov, N. V. Kuznetsov, O. A. Kuznetsova, S. M. Seldedzhi, and V. I. Vagaitsev, "Hidden oscillations in dynamical systems," Transaction on Systems and Control, vol. 6, pp. 54-67, 2011.

[34] G. A. Leonov, N. V. Kuznetsov, and V. I. Vagaitsev, "Localization of hidden Chua's attractors," Physics Letters. A, vol. 375, no. 23, pp. 2230-2233, 2011.

[35] G. A. Leonov, N. V. Kuznetsov, and V. I. Vagaitsev, "Hidden attractor in smooth Chua systems," Physica D, vol. 241, no. 18, pp. 1482-1486, 2012. 
[36] G. A. Leonov and N. V. Kuznetsov, "Hidden attractors in dynamical systems. From hidden oscillations in HilbertKolmogorov, Aizerman, and Kalman problems to hidden chaotic attractor in Chua circuits," International Journal of Bifurcation and Chaos, vol. 23, no. 1, Article ID 1330002, 2013.

[37] S. Brezetskyi, D. Dudkowski, and T. Kapitaniak, "Rare and hidden attractors in Van der Pol-Duffng oscillators," European Physical Journal: Special Topics, vol. 224, no. 8, pp. 1459-1467, 2015.

[38] T. Kapitaniak and G. A. Leonov, "Multistability: uncovering hidden attractors," European Physical Journal: Special Topics, vol. 224, no. 8, pp. 1405-1408, 2015.

[39] D. Dudkowski, S. Jafari, T. Kapitaniak, N. V. Kuznetsov, G. A. Leonov, and A. Prasad, "Hidden attractors in dynamical systems," Physics Reports, vol. 637, pp. 1-50, 2016.

[40] P. R. Sharma, M. D. Shrimali, A. Prasad, N. V. Kuznetsov, and G. A. Leonov, "Control of multistability in hidden attractors," European Physical Journal: Special Topics, vol. 224, no. 8, pp. 1485-1491, 2015.

[41] Z. T. Zhusubaliyev, E. Mosekilde, A. N. Churilov, and A. Medvedev, "Multistability and hidden attractors in an impulsive Goodwin oscillator with time delay," European Physical Journal: Special Topics, vol. 224, no. 8, pp. 1519-1539, 2015.

[42] Z. T. Zhusubaliyev and E. Mosekilde, "Multistability and hidden attractors in a multilevel DC/DC converter," Mathematics and Computers in Simulation, vol. 109, pp. 32-45, 2015.

[43] A. Wolf, J. B. Swift, H. L. Swinney, and J. A. Vastano, "Determining Lyapunov exponents from a time series," Physica D, vol. 16, no. 3, pp. 285-317, 1985.

[44] N. V. Kuznetsov, "The Lyapunov dimension and its estimation via the Leonov method," Physics Letters A, vol. 380, no. 25-26, pp. 2142-2149, 2016.

[45] N. V. Kuznetsov, T. A. Alexeeva, and G. A. Leonov, "Invariance of Lyapunov exponents and Lyapunov dimension for regular and irregular linearizations," Nonlinear Dynamics, vol. 85, no. 1, pp. 195-201, 2016.

[46] G. A. Leonov, N. V. Kuznetsov, N. A. Korzhemanova, and D. V. Kusakin, "Lyapunov dimension formula for the global attractor of the Lorenz system," Communications in Nonlinear Science and Numerical Simulation, vol. 41, pp. 84-103, 2016.

[47] S. Bouali, A. Buscarino, L. Fortuna, M. Frasca, and L. V. Gambuzza, "Emulating complex business cycles by using an electronic analogue," Nonlinear Analysis. Real World Applications, vol. 13, no. 6, pp. 2459-2465, 2012.

[48] W.-J. Zhou, Z.-P. Wang, M.-W. Wu, W.-H. Zheng, and J.-F. Weng, "Dynamics analysis and circuit implementation of a new three-dimensional chaotic system," Optik, vol. 126, no. 7-8, pp. 765-768, 2015.

[49] Q. Lai and L. Wang, "Chaos, bifurcation, coexisting attractors and circuit design of a three-dimensional continuous autonomous system," Optik, vol. 127, no. 13, pp. 5400-5406, 2016.

[50] L. Fortuna, M. Frasca, and M. G. Xibilia, Chua's Circuit Implementation: Yesterday, Today and Tomorrow, World Scientific, Singapore, 2009.

[51] G. Bianchi, N. V. Kuznetsov, G. A. Leonov, M. V. Yuldashev, and R. V. Yuldashev, "Limitations of PLL simulation: hidden oscillations in MatLab and SPICE," in Proceedings of the 7th International Congress on Ultra Modern Telecommunications and Control Systems and Workshops (ICUMT '15), pp. 79-84, October 2015.
[52] G. Bianchi, N. V. Kuznetsov, G. A. Leonov, S. M. Seledzhi, M. V. Yuldashev, and R. V. Yuldashev, "Hidden oscillations in SPICE simulation of two-phase Costas loop with non-linear VCO," IFAC-PapersOnLine, vol. 49, no. 14, pp. 45-50, 2016.

[53] L. M. Pecora and T. L. Carroll, "Synchronization in chaotic systems," Physical Review Letters, vol. 64, no. 8, pp. 821-824, 1990.

[54] S. Vaidyanathan and S. Rasappan, "Global chaos synchronization of n-scroll Chua circuit and Lur'e system using backstepping control design with recursive feedback," Arabian Journal for Science and Engineering, vol. 39, no. 4, pp. 3351-3364, 2014.

[55] A. Ouannas, A. T. Azar, and R. Abu-Saris, "A new type of hybrid synchronization between arbitrary hyperchaotic maps," International Journal of Machine Learning and Cybernetics, pp. $1-8,2016$.

[56] A. Stefanski, P. Perlikowski, and T. Kapitaniak, "Ragged synchronizability of coupled oscillators," Physical Review E, vol. 75, no. 1, Article ID 016210, 2007.

[57] S. Boccaletti, J. Kurths, G. Osipov, D. L. Valladares, and C. S. Zhou, "The synchronization of chaotic systems," Physics Reports A, vol. 366, no. 1-2, pp. 1-101, 2002.

[58] L. Fortuna and M. Frasca, "Experimental synchronization of single-transistor-based chaotic circuits," Chaos, vol. 17, no. 4, Article ID 043118, 2007.

[59] H. K. Khalil, Nonlinear Systems, Prentice Hall, New Jersey, NJ, USA, 3rd edition, 2002. 


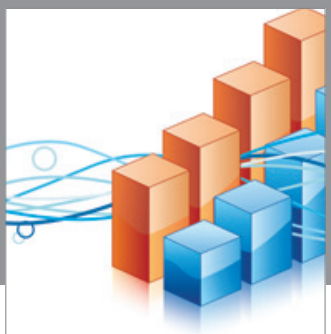

Advances in

Operations Research

vatem alat4

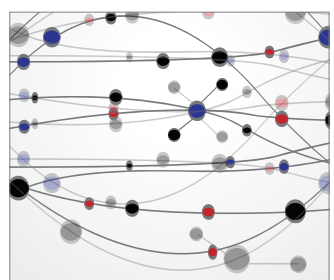

\section{The Scientific} World Journal
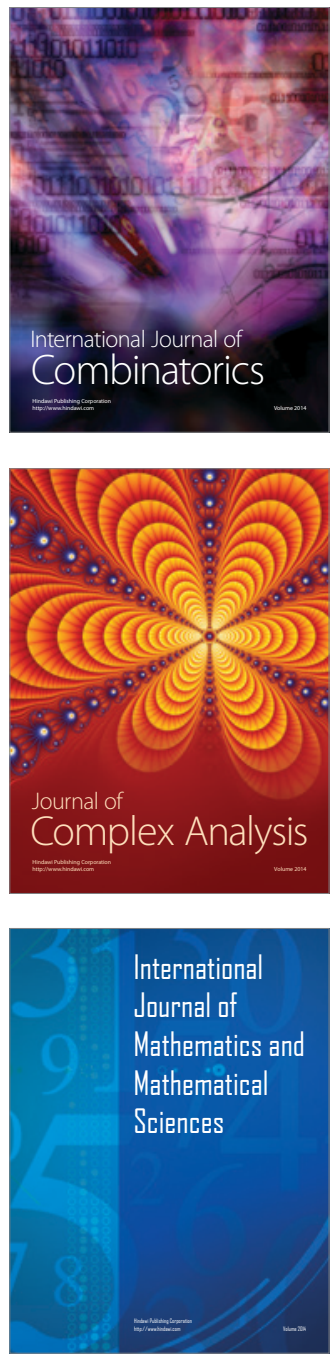
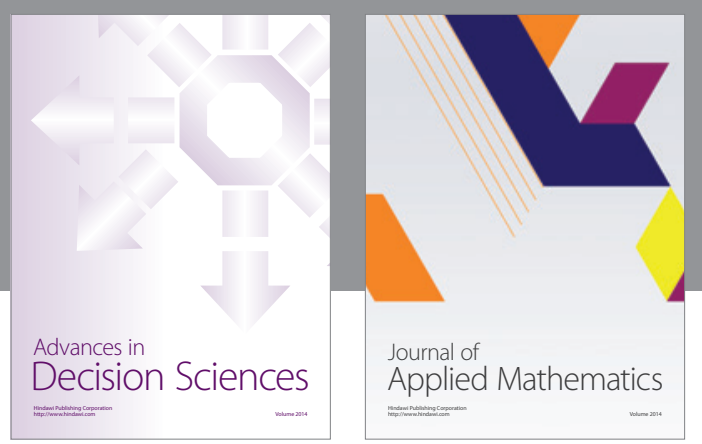

Algebra

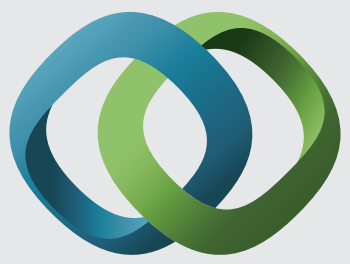

\section{Hindawi}

Submit your manuscripts at

https://www.hindawi.com
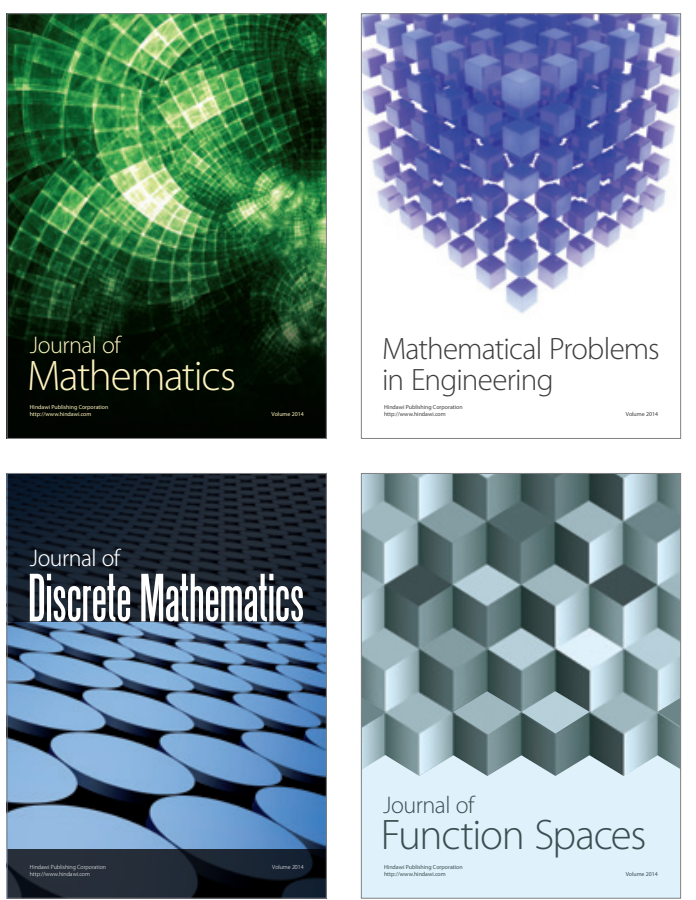

Mathematical Problems in Engineering
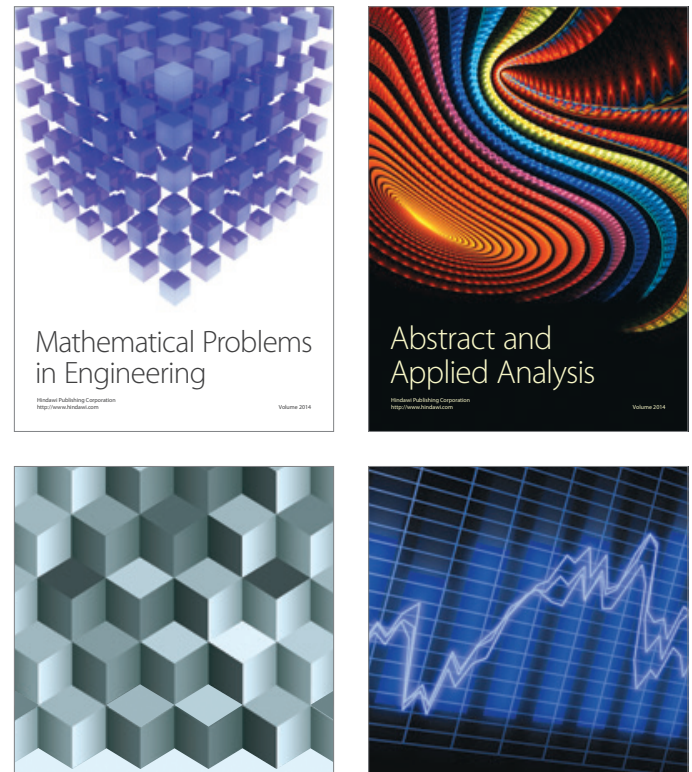

Journal of

Function Spaces

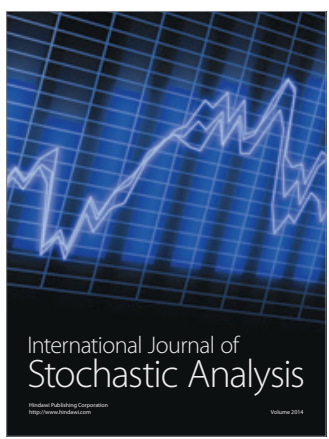

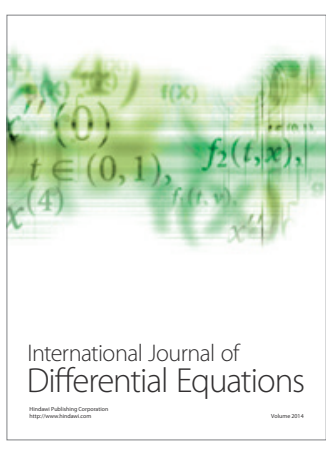
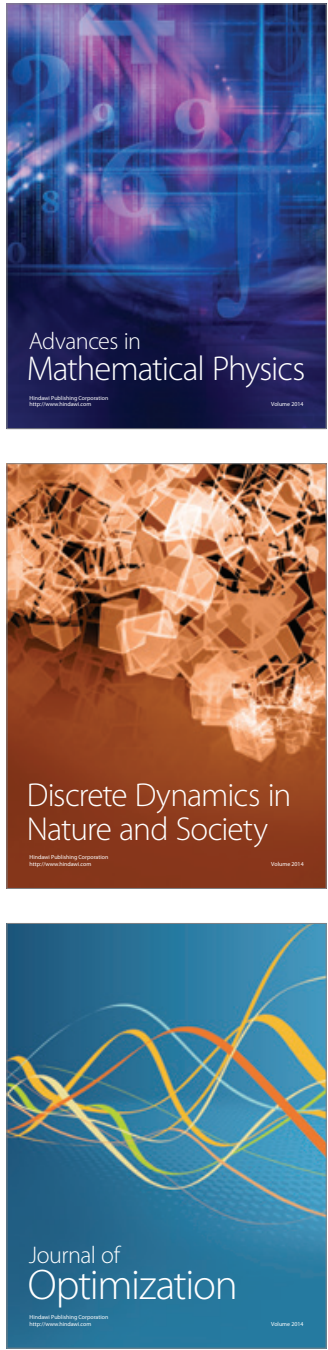